\title{
A three-dimensional optical interconnection architecture with minimal growth rate of system size
}

\author{
Haldun M. Ozaktas, Yaakov Amitai and Joseph W. Goodman \\ Information Systems Laboratory, Department of Electrical Engineering, Stanford University, Stanford, CA 94305, USA
}

Received 28 March 1991; revised manuscript received 30 April 1991

\begin{abstract}
We present a three-dimensional optical interconnection architecture that can potentially approach the least possible system size of any architecture within a numerical factor of the order of $\sim 10$. This architecture can provide an arbitrary pattern of connections among a three-dimensional array of points.
\end{abstract}

\section{Introduction}

In a previous paper [1] we compared the growth rate of system size for various optical interconnection architectures and presented an architecture that can potentially approach the least possible system size of any two-dimensional optical architecture, both in terms of growth rate and numerical constants. This architecture could provide an arbitrary pattern of connections among a two-dimensional array of points. In this paper we describe a three-dimensional version of this architecture.

\section{System size considerations}

We assume that we are to provide an arbitrary pattern of $k N$ pairwise connections among the $2 k N$ terminals of $N$ computing elements (which may be electronic chips) with $2 k$ optical terminals each. For simplicity the extension to fan-out is not considered. The elements will be laid out on a three-dimensional regular cartesian grid with (as yet undetermined) lattice spacing $d$. A significant parameter of such a layout is its average connection length $l$, where $l$ denotes the distance of a particular connection and the overbar denotes averaging over all connections. This quantity may be expressed as $\Gamma=\kappa N^{q-2 / 3} d$. (This is the three-dimensional version of the results given in ref. [2] for two dimensions.) $\kappa$ is a coefficient which is often of the order of unity. $q$ is a measure of the connectivity of the system and satisfics $2 / 3 \leq q \leq 1$.

The minimum size of such a system may be limited by several considerations. In this paper we assume that the size of the system is determined by the space necessary to provide communication among the elements, and that other considerations, such as the size of the elements or heat removal, are not limiting factors.

The least possible system linear extent $L$ for a threedimensional optical system is given by [1]

$L=N^{1 / 3} d=(k \kappa)^{1 / 2} N^{1 / 2} \tilde{\mathscr{F}} \lambda$,

where $\bar{F}$ is a constant factor which can be as small as the order of unity for a diffraction limited system but may be quite larger in practice. This growth rate of system size can be approached by the use of discrete fibers of diameter $\sim \mathscr{F} \lambda$. (To see this, note that the volume occupied by the $k N$ interconnections is given by $k N I(\mathscr{F} \lambda)^{2}$. Equating this to $N d^{3}$, the total available volume, it is easy to show that $L=N^{1 / 3} d$ $\left.=(\mathrm{k} \kappa)^{1 / 2} N^{q / 2} \mathscr{F} \lambda\right)$. However, the value of $\mathscr{F}$ would be very large in this case (probably $>100$ ), so that although the least possible growth rate is achieved, we would nevertheless end up with a large system. It is desirable to find architectures which achieve the least possible system size numerically, as well as in terms of growth rate [3]. In the next section we describe an architecture that may come as much as an 
order of magnitude close to the best possible.

\section{The three-dimensional multi-facet architecture}

The system is constructed in the form of a sandwich of a large number of layers, as illustrated in fig. 1. The layers are separated by glass slabs. There are two kinds of layers. There are $N^{1 / 3}$ elements layers, on which $N^{1 / 3} \times N^{1 / 3}$ elements are arrayed in cartesian manner. (In the figure, we choose $N=3^{3}$ for illustration. In practice $N$ will probably be much larger.) Between the element layers are an as yet unspecified number of holographic emulsion layers, spaced at a small distance $h$ from each other. The holograms in these layers can be fabricated directly as computer generated holograms (CGHs) [4], can be recorded holographically as computer originated holograms [5] or can be recorded recursively from other aspherical holograms [6]. Ultimately, in the more distant future, it may be possible to fabricate the whole system (including electronics, transducers, and holograms) monolithically with MBElike techniques. The height of the system is equal to its lateral linear extent $L$, forming a perfect cube, so that there are $\simeq L / h$ emulsion layers, assuming the elements are not very thick. The average refractive index of the emulsion material is equal to that of the glass slabs.

Each connection is established in at most six hops, using five holograms. The path of light for a connection between two transducers situated on elements located at diametrically opposite corners of the cube is symbolically depicted in fig. 2 . The diameters of holograms $\mathscr{H}_{\mathrm{s}}$ and $\mathscr{H}_{\mathrm{r}}$, which we denote by $d_{\mathrm{s}}$ and $d_{\mathrm{r}}$ respectively, are independent of the length of the connection. Both $d_{\mathrm{s}}$ and $d_{\mathrm{r}}$ should be chosen as small as possible, however $d_{\mathrm{s}}$ must be chosen consistent with the $f$-number of the source and the distance of $\mathscr{H}_{\mathrm{s}}$ to the source. Most probably, the holograms $\mathscr{H}_{\mathrm{s}}$ and $\mathscr{H}_{\mathrm{r}}$ would be located immediately above the sources and detectors. The diameters of holograms $\mathscr{H}_{\mathrm{a}}\left(d_{\mathrm{a}}\right), \mathscr{H}_{\mathrm{b}}\left(d_{\mathrm{b}}\right)$ and $\mathscr{H}_{\mathrm{c}}\left(d_{\mathrm{c}}\right)$ are chosen according to the length of the connection. Larger holograms will be used for longer connections so as to compensate for the effects of diffraction.

For many connections, all six hops may not be necessary. However, the above scheme ensures that a connection between any two elements can be established regardless of their relative position. Several other schemes likewise employing a bounded number of holograms regardless of distance are also possible. The crucial point is that the line-of-sight problem can be overcome by breaking the connection into a bounded number of hops whose total path length does not exceed the euclidean distance between the two elements to be connected by more than a bounded geometrical factor of the order of unity. It is important to keep the number of hops bounded with increasing number of elements and system size so that the interconnect efficiency does not decrease with increasing $N$. For instance, if each hologram can be fabricated with $\geq 95 \%$ diffraction efficiency, the interconnect efficiency will be $\geq 75 \%$ regardless of $N$. Also note that there is great freedom in choosing

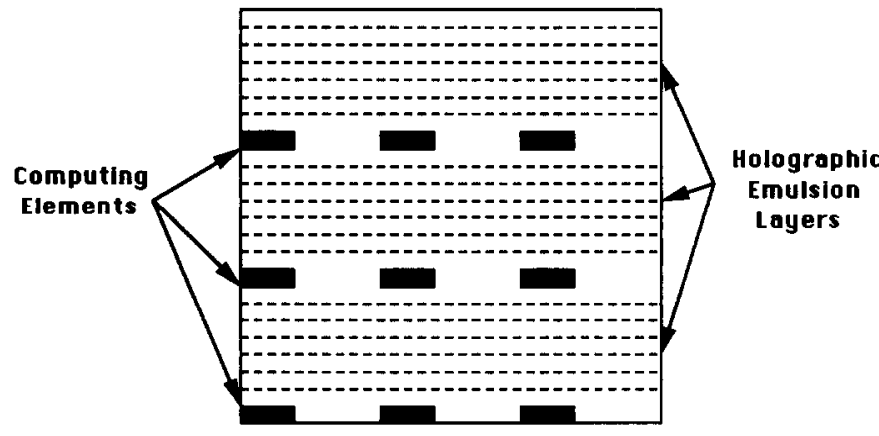

a. Side Uiew

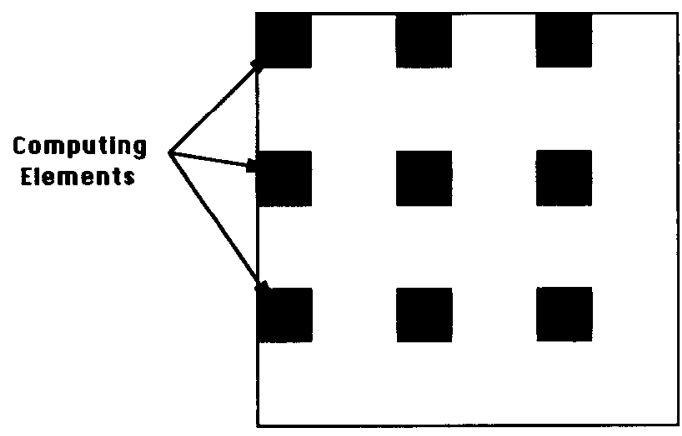

b. Top View

Fig. 1. The three-dimensional multi-facet architecture. a. Side view. b. Top view. 

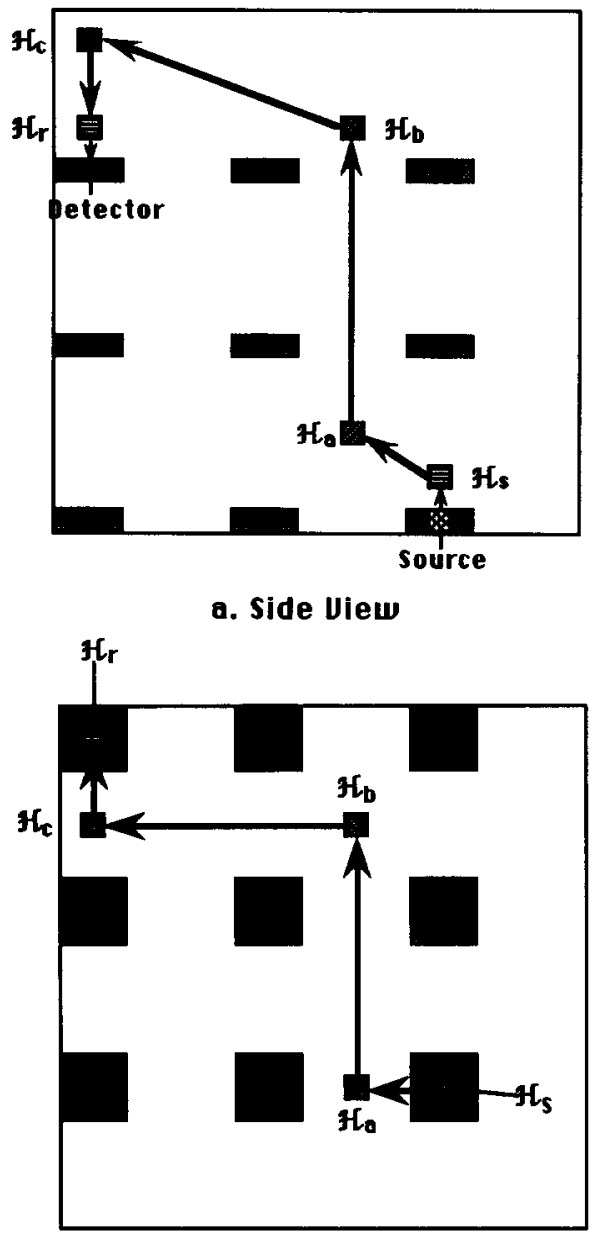

b. Top View

Fig. 2. A connection between two optical transducers situated on elements located at diametrically opposite corners of the system. a. Side View, b. Top view.

the paths and hologram locations for the various connections.

Since there are $L / h$ emulsion layers, the total area available for the holograms is $L^{2} \times L / h=L^{3} / h$. Equating this to the area required for the five holograms associated with each of the $k N$ connections yields

$$
L^{3} / h=k N \overline{\left(d_{\mathrm{s}}^{2}+d_{\mathrm{a}}^{2}(l)+d_{\mathrm{b}}^{2}(l)+d_{\mathrm{c}}^{2}(l)+d_{\mathrm{r}}^{2}\right)},
$$

where the overbar denotcs averaging over all connections. The area of any two consecutive holograms $\mathscr{H}_{x}$ and $\mathscr{H}_{v}$ must be chosen such that $d_{x}=\mathscr{F} \lambda l_{x y} / d_{y}$ where $l_{x y}$ is the path length between them. $l_{x y}$ will not be different than the euclidean distance between the elements by more than a numerical factor of the order of unity. Ignoring such factors to simplify our analysis, let us take $d_{\mathrm{a}}^{2}=d_{\mathrm{b}}^{2}=d_{\mathrm{c}}^{2} \simeq \mathscr{F} \lambda l$ for all holograms and also assume $d_{\mathrm{s}}=d_{\mathrm{r}}=$ constant. As mentioned previously, we are using larger holograms for longer connections. Then, within a numerical factor of the order of unity,

$L^{3} / h \simeq k N\left(d_{\mathrm{s}}^{2}+\mathscr{F} \lambda k N^{q-1} L\right)$,

since

$\left[=\kappa N^{q-2 / 3} d=\kappa N^{q-2 / 3} L / N^{1 / 3}=\kappa N^{q-1} L\right.$.

Thus the system linear extent is found to satisfy

$L \simeq\left(k d_{\mathrm{s}}^{2} h\right)^{1 / 3} N^{1 / 3}+(k \kappa)^{1 / 2} N^{q / 2} \sqrt{(\mathscr{F} \lambda) h}$.

which becomes for $q>2 / 3$ and large $N$

$L \simeq(k \kappa)^{1 / 2} N^{/ 2} \sqrt{(\mathscr{F} \lambda) h}$,

which exhibits the least possible growth rate of any three-dimensional system $\propto N^{1 / 2}$. Numerically, we are worse off than the smallest possible system size $(k \kappa)^{1 / 2} N^{q / 2} \mathscr{F} \lambda$ by a factor of $\sqrt{h / \mathscr{F}} \lambda \geq 1$. If $\mathscr{F} \sim 1$, $\lambda \sim 1 \mu \mathrm{m}$, and $h \sim 100 \mu \mathrm{m}$, then we are doing about an order of magnitude worse than the best possible. As an example, the linear extent $L$ of a system with $N=10^{6}, k=50$ and $q=0.8$ would be of the order of ten centimeters.

As in the two-dimensional folded multi-facet architecture [1], care must be exercised so that the wave vector of any propagating beam does not unintentionally fall on the Bragg cone of a hologram it is supposed to pass through without interaction. Given that there is a very large degree of freedom at our disposal in choosing the locations of the holograms for each connection, this should be easily overcome. It is possible to envision a computer aided design procedure that would determine the optimal locations for each connection.

\section{Conclusion}

The problem of optically interconnecting an array of points can be viewed as an imaging problem. Conceptually, a number of transducers can be imaged onto their target detectors by tailoring the refractive index distribution $n(x, y, z)$ of the medium between 
them. In other words, an optically interconnected computer may be viewed as a volume hologram with the computing elements embedded into it.

In this paper, we have presented a threc-dimensional optical interconnection architecture which can achieve the least possible growth rate of system size of any interconnection architecture with increasing number of elements. Numerically, our architecture may approach the best possible within about an order of magnitude, depending on how small the spacing between the emulsion layers can be made.

In this paper we have not discussed issues such as power distribution to the computing elements, heat removal from system, noise etc. which must be addressed prior to the actual construction of such a system. Considerations of these issues are beyond the scope of this preliminary study and are areas for future research.

\section{Acknowledgement}

This work was supported by the Air Force Office of Scientific Research under Grant No. AFOSR-880024.

\section{References}

[1] H.M. Ozaktas, Y. Amitai and J.W. Goodman, Optics Comm. 82 (1991) 225.

[2] M. Feuer, IEEE Transactions on Computers 31 (1982) 29.

[3] H.M. Ozaktas and J.W. Goodman, J. Opt. Soc. Am. A 7 (1990) 2100.

[4] W.H. Lee, Progress in Optics 16 (1978) 119.

[5] Y. Amitai and J.W. Goodman, Optics Comm. 80 (1990) 107.

[6] Y. Amitai and J.W. Goodman, Design of substrate mode holographic interconnects with different recording and readout wavelengths, Appl. Optics, in print. 